\title{
Physics Formalism Helmholtz Matrix to Coulomb Gage
}

\author{
Rajan Iyer
}

\section{Email address: engginc@msn.com}

\section{Environmental Materials Theoretical Physicist, Department of Physical Mathematics Sciences Engineering Project Technologies, Engineeringinc International Operational Teknet Earth Global, Tempe, Arizona, United States of America}

\begin{abstract}
Iyer Markoulakis Helmholtz Hamiltonian mechanics formalisms mathematically modeled physics with vortex rotational fields acting with gradient fields, typically in zero-point microblackhole general fields. Here, Helmholtz metrics have been gaged to Coulombic Hilbert metrics, representing Gilbertian and Amperian natures of electromagnetic fields out of the mechanical fields from Helmholtz Hamiltonian mechanics, extending to vacuum gravitational fields gage.
\end{abstract}

This ansatz general gaging helps to properly isolate field effects with physical analyses mechanical, electric, magnetic components within the analytical processes. Vacuum gravitational fields and the flavor Higgs-Boson matter inertial gravitational fields have been thus quantified extending to stringmetrics constructs matrix showing charge asymmetry gage metrics, having the power to analyze dark energy superluminal phase, dark matter luminal phase, and light matter subluminal phase. Interpreting particle physics gage matrix pointing to Dirac seas of electrons, monopoles with positrons, electron-positron annihilation leading to energy production, and the relativistic energy generating matter. Physical analysis systems with switching modes $\{0$, off, on $\}$ are mathematically characterized in terms of functor $\{0$ mode, on_mode $\}$ with off_mode providing compatible coupling function to activate functional $\{0$ mode, on_mode $\}$. These processes maybe causality to quantum entangle categorical mode states, with default states of 0 and on modes having quantum decoherence.

Quantum ASTROPHYSICS gage metrix analyzes superluminal profile of signals velocity generating electron-positron chain like "curdling" action, that is consistent with physics literature reporting the nature of electron-photon observed oscillatory fields configurations. This then helps proposing mechanism of creation of neutrino antineutrino pair orthogonal to electron positron "curdling" planes, that may lead to formation of protonic hydrogen of stars or orthogonally muon particles. These aspects will help to explain receding or fast expanding universe with the dark matter in terms of flavor metrics versus gage associating metrics fields. Vacuum and gravitational monopoles, that are representation of compressed mass out of vortex Helmholtz decomposition fields have been interpolated, consistent with physics literature, with vacuum monopoles proposed to occur at cosmos extent of infinity. 
Key Words: Physics, Helmholtz Hamiltonian Mechanics, Matrix Algebra, Electromagnetism, Gravitational, Gage, Coulomb, Switches, Modeling Parameters, Transforms, Quantum Astrophysics, Signal gaging, stringmetrics.

\section{Introduction:}

Gage transforms are necessary part of physics today to match relativity with quantum physics, developing consistency to observables classically, where all the basic forces are unified at the scale set by gauge-coupling unification quantitatively, thus for example, explaining observed feebleness of gravity [1]. Gauge fields are included in the Lagrangian to ensure its invariance under the local group transformations, also called gauge invariance $[2,3,4,5,6]$. Typical classical Maxwellian electrodynamics have gauge fields, that are like equation 41 in [7]. Gauge theory, a class of quantum field theory, a mathematical theory involving both quantum mechanics and Einstein's special theory of relativity that is commonly used to describe subatomic particles and their associated wave fields may also constitute scalar gauge fields [8]. In author's original conceptuality, gage invariance is like removing variables using high differentiation to get to constants, that will have consistent values across the board of all types mechanical, electronic, and magnetic fields.

Typical gauge transformation in general can be any formal, systematic transformation of the potentials that leaves the fields invariant, although in quantum theory it can be perhaps a bit more subtle than that because of the additional degree of freedom represented by the quantum phase $[8,9]$. These gauge transformations between possible gauges tend to form a Lie group, in general referred as symmetry group or the gauge group of the theory; Lie algebra of group generators quantifies a lie group [9].

Gage conversions are quite useful to invariantly transfer information of fields of one type, like mechanics onto the fields of another type, like electromagnetism, as above literature suggests. For example, Helmholtz Hamiltonian mechanics metrics quantifying mechanical fields can be gaged to Coulombic Hilbert metrics, representing Gilbertian and Amperian natures of electromagnetic fields $[6,21,22]$.

Iyer Markoulakis Helmholtz Hamiltonian mechanics formalisms mathematically modeled the physics with vortex rotational fields acting in cohort to gradient fields, typically in a zero-point as well as of microblackhole general fields, modelled by abstracting observations with Ferrolens of vortex magnetic fields within a real magneton configured to synthetic magnetic monopole assembly $[10,11]$. Author has extensively successfully applied this ansatz formalism to general also specific problem solving of attractive, repulsive forces specifically encountered in all electronic and magnetic entity forms, that will include monopoles within a dipole quagmire, performing physical analysis specifically concentrating on the process physics [12].

Here, ansatz formalism quantifies gage to electromagnetic fields mathematically. Section 2 models mathematically with Theoretical Results Physics Gaging Formalism showing 
construction of gage matrices fundamentally from field and energy forms to gage metrics by applying original micro macro connectivity formalism author has recently developed [13] with Section 2.1 modeling configuring constructs that will abstract derivative formalism of Helmholtz Hamiltonian mechanism partial differential equation sets already developed earlier with author working with experimental physicist, formulating Iyer Markoulakis metrics general formalism [11]. Section 3 expounds Physical Analysis with Results and Discussions. Section 3.1 shows with a brief note about how Helmholtz metrics will gage to axial eccentric quantum fields gravity. Section 3.2 superimposes conceptually model of Signal Superluminal Profile Graph onto Proper Real Extended Time. Section 3.3 applies ansatz gage metrix formalism to Particle Physics Gage Matrix to model a way to quantify Helmholtz Hamiltonian Mechanics to Dirac Monopoles. Section 3.4 shows Quantum Astrophysics Gage Matrix conceptualizations, then Section 3.5 extends formalism with interpretations on virtual gravitational dipoles, as well as newly developing concept gravitational monopoles at Planck dimensions. Section 4 summarizes modeling ansatz gage general formalisms with application to unitarization, linking to special unitary groups.

\section{Theoretical Results Physics Gaging Formalism}

Problem: Hamiltonian Schrodinger wave function is not a gage invariant Lagrangian, hence it will have to undergo transformation also called gauge transformation in this context obtaining the unitary equivalent Schrödinger equation [14]; per author's explainable that will constitute essentially acting like functor, i. e. physically distinct categories.

Hint literature solving problem: In recent years, functor - category of cobordisms - extending topological quantum field theory (TQFT) has been completely formalized with action functional that generates geometrically quantized models [15]. Schrodinger equation will be invariant under a gauge transformation, by changing the wavefunction by a phase $\mathrm{e}^{\mathrm{ie} \Lambda / \hbar c}[16]$, acting like a function that generate functional coupling categories. \{In Schwarz-type TQFTs, the correlation functions or partition functions of a system are computed by the path integral of metric-independent action functionals. Example, for time-dependent correlation functions, the time-ordering operator is included; these typical correlation functions are also simply called correlators. The correlation function can also be interpreted physically as the amplitude for propagation of a particle or excitation between $y$ and $x$ parameters, having like equations with product of wave functions [17]. The Fock complex vertex operator implements the aspects of those interactions in the BRST-BV formulation of the theory, for example [18]. The Fock space special unitary group built upon the spin 0 ground state too then gets a natural grading by spin [19]. Fock space may be considered as an algebraic construction used in quantum mechanics to construct the quantum states space of a variable or unknown number of identical particles from a single particle Hilbert space; also, one may infer informally, a Fock space is the sum of a set of Hilbert spaces representing zero particle states, one particle states, two particle states, and so on [20]. If the identical particles are bosons, the $n$-particle states are vectors in a symmetrized tensor product of $n$ single-particle Hilbert spaces; for example, if the identical particles are fermions, the $n$-particle states are vectors in an antisymmetrized tensor product of $n$ single-particle Hilbert spaces; also, technically that Fock space is the Hilbert space 
completion of the direct sum of the symmetric or antisymmetric tensors in the tensor powers of a single-particle Hilbert space $[19,20]$.

Solution: Applying above "Hint literature", having a coupling function to the quantum wave function may enable transforming of a typical functor, which is mathematically equivalently logic of physically distinct categories, compatibly to gage functional, that will equivalently make tensor or vector to scalar. To achieve this, author has developed matrix process general formalism with Equation (11) [13]: $\mathrm{F}_{\mathrm{t}}^{\mathrm{E}}=\boldsymbol{\rho}(\mathrm{t})\left(\left\langle\boldsymbol{\Psi}_{\mu}(\mathrm{t})\right| \boldsymbol{\Psi}^{\mu}(\mathrm{t})\right)^{-1} \mathbf{V}$ that can be graphed by setting $\mathrm{Y}=$ $f(X)$, with $X=\rho(t)$ and $Y=F_{t}^{E}$, representing observables' functional commutator varying with quantum density matrix, $\rho(t)$ characterizing pure state, like coupling constant of general relativity. From these, we can interpret that $f$, the function operator transforms micro to macro parametrically quantum density matrix, $\rho(t)$ to functional commutator, $\mathrm{F}^{\mathrm{E}} \mathrm{t}$, with inner product of up and down aspects of vortex action wave eigenfunctions, $\boldsymbol{\Psi}_{\mu}(\mathrm{t})$ and $\boldsymbol{\Psi}^{\mu}(\mathrm{t})$, acting alongside general energy fields, $\mathrm{V}$, like scalar potential in general relativity. Author has also inferred that effectively, quantum density matrix, $\rho(t)$ can be seen to be influencing time event process via energy quanta, wherein time fields that are typical of microblackholes analytically are extractable from differential equations (43) and (46) developed and presented all with in modeling lyer Markoulis formalism [11] characterizing these processes. Notable is also that outliers with $X-Y$ plot of real data may provide observables of monopoles that may be measurable systematically with physical analysis, such as observables measured experimentally in BoseEinstein condensates as well as within experimental measurements of monopoles using spin ice specifics $[11,12]$. Author will exemplify these further in subsequent analysis here, applying this formalism enabling gaging of Helmholtz Hamiltonian mechanics to electromagnetic quantum fields, following transformation of Helmholtz metrics via Coulomb gage in Section 2.1.

\subsection{Configuring Abstraction through Derivative Formalism from Iyer Markoulakis Helmholtz Hamiltonian to the Amperian Gilbertian Hilbert Coulomb Matrix Gage}

Originally, Helmholtz matrix operating Density Field Matrix Eigenvector Operators per magneton $[10,11]$ observations lead to physical mathematical quantum constructs $[11,12]$ that shows the Helmholtz matrix equated to gage parameters:

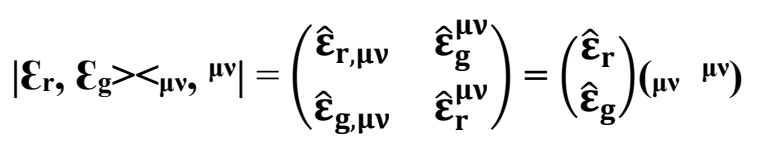

with $\widehat{\boldsymbol{\varepsilon}}_{\mathbf{r}, \boldsymbol{\mu \nu}}=0, \hat{\boldsymbol{\varepsilon}}_{\mathbf{r}}^{\boldsymbol{\mu \nu}}=\widehat{\mathbf{M}}$, for rotational vortex fields, then $\widehat{\boldsymbol{\varepsilon}}_{\mathbf{g}}^{\boldsymbol{\mu \nu}}=\widehat{\mathbf{G}}, \& \widehat{\boldsymbol{\varepsilon}}_{\mathbf{g}, \boldsymbol{\mu \nu}}=\widehat{\mathbf{G}}^{-\mathbf{1}}$, and for gradient fields, according to gauge and non-gauge field tensor operationally. $\mathbf{M}$ s are Hilbert gauge like Higgs metrics mass of Higgs-Boson matter, while $\widehat{\mathbf{G}}$ is the Coulomb gage equivalent fields representation, with the $\widehat{\mathbf{G}}^{\mathbf{- 1}}$ is the Coulomb reverse equivalent fields representing vortex.

Partial differential equations characterizing zero-point microblackhole entities Hamiltonian operator physics have been developed elsewhere $[11,12]$ : 
(i) Zero_point Hamiltonian operator eigen fields tensor zero_point gradient differential equations energy gradient fields are given by [11]:

$\nabla^{3} \mathbf{E}_{\mathrm{g}}{ }^{\mu v} \cdot \nabla^{2} \mathbf{E}_{\mathrm{g}, \mu v}=[(2-\mathrm{ich}) / \mathrm{ich}] \nabla^{3} \mathbf{E}_{\mathrm{g}, \mu v} \cdot \nabla^{2} \mathbf{E}_{\mathrm{g}}{ }^{\mu v}$

(ii) microblackhole Hamiltonian operator eigen fields rotational tensor microblackhole differential equations with Helmholtz rotational fields are given by [11]:

$\nabla^{2} \boldsymbol{\varepsilon}_{\mathbf{r}, \mu v}-\left\{\mathrm{i}\left(\mathrm{t}_{\mathrm{f}}-\mathrm{t}_{\mathrm{i}}\right) / \hbar\right\}\left[\boldsymbol{\varepsilon}_{\mathbf{r}, \mu v}\left(1+\ln \left|\boldsymbol{\varepsilon}_{\mathbf{r}, \mu v}\right|\right]^{-1}\left(\nabla \boldsymbol{\varepsilon}_{\mathbf{r}, \mu v}\right)^{2}+\left\{\mathrm{i}\left(\mathrm{t}_{\mathrm{f}}-\mathrm{t}_{\mathrm{i}}\right) / \hbar\right\}\left[\boldsymbol{E}_{\mathbf{r}, \mu v} /\left(1+\ln \left|\boldsymbol{\varepsilon}_{\mathbf{r}, \mu v}\right|\right]=0\right.\right.$

$\nabla^{2} \boldsymbol{E}_{\mathbf{r}}{ }^{\mu v}-\left\{\mathrm{i}\left(\mathrm{t}_{\mathrm{f}}-\mathrm{t}_{\mathrm{i}}\right) / \hbar\right\}\left[\boldsymbol{E}_{\mathbf{r}}{ }^{\mu v}\left(1+\ln \left|\boldsymbol{E}_{\mathbf{r}^{\mu v}}\right|\right]^{-1}\left(\nabla \boldsymbol{E}_{\mathbf{r}}{ }^{\mu v}\right)^{2}+\left\{\mathrm{i}\left(\mathrm{t}_{\mathrm{f}}-\mathrm{t}_{\mathrm{i}}\right) / \hbar\right\}\left[\boldsymbol{E}_{\mathbf{r}}{ }^{\mu v} /\left(1+\ln \left|\boldsymbol{E}_{\mathbf{r}^{\mu v}}\right|\right]=0\right.\right.$

To conform with system of P.D.E.s, $\hat{\boldsymbol{\varepsilon}}_{\mathrm{g}}$ must be transformed to the energy form characteristics like: $\widehat{\boldsymbol{\varepsilon}}_{\mathbf{g}}:=>\mathbf{E}_{\mathbf{g}}$; however, $\widehat{\boldsymbol{\varepsilon}}_{\mathbf{r}}$ must be in field forms $\widehat{\boldsymbol{\varepsilon}}_{\mathbf{r}}:=>\widehat{\boldsymbol{\varepsilon}}_{\mathbf{r}}$. Therefore, applying Equations (2), (3.1), \& (3.2), Equation (1) equivalent Helmholtz matrix will have characteristics:

$\left(\begin{array}{ll}\hat{\varepsilon}_{\mathrm{r}, \mu \nu} & \hat{\varepsilon}_{\mathrm{g}}^{\mu v} \\ \hat{\varepsilon}_{\mathrm{g}, \mu \nu} & \hat{\varepsilon}_{\mathrm{r}}^{\mu \nu}\end{array}\right)=>::<=\left(\begin{array}{cc}\hat{\varepsilon}_{\mathrm{r}, \mu \nu} & \nabla^{2} \mathbf{E}_{\mathrm{g}}^{\mu \nu} \\ \nabla^{2} \mathbf{E}_{\mathrm{g}, \mu \nu} & \hat{\varepsilon}_{\mathrm{r}}^{\mu \nu}\end{array}\right)$

Note: It is possible to transform from Helmholtz metrics, using Coulomb gage that will link to Coulomb branch gage group with Hilbert series having SuperSymmetry (SUSY) Quantum Field Theory (QFT) charge conjugation $[21,22]$. Subsequent to that it is possible to link charge conjugation to rotating charges per Dirac Maxwell Einstein Kerr Newmann metrics [23, 24].

Based on the arguments $[11,12,21-24]$ and above explanations, we transform Helmholtz matrix Equation (4) onto gage matrix, i.e. we convert Helmholtz to gauge by having equivalently Helmholtz metrics to Coulomb gage: $\left\{\boldsymbol{\nabla}^{\mathbf{2}} \mathbf{E}_{\mathbf{g}, \boldsymbol{\mu \nu}}, \boldsymbol{\nabla}^{\mathbf{2}} \mathbf{E}_{\mathbf{g}}^{\boldsymbol{\mu \nu}}\right\} \mid=>\left\{\widehat{\mathbf{G}}_{\mathbf{g}, \boldsymbol{\mu \nu}}, \widehat{\mathbf{G}}_{\mathbf{g}}^{\boldsymbol{\mu \nu}}\right\}$, with branching to Hilbert gauge $\left\{\hat{\boldsymbol{\varepsilon}}_{\mathbf{r}, \boldsymbol{\mu \nu}}, \hat{\boldsymbol{\varepsilon}}_{\mathbf{r}}^{\boldsymbol{\mu \nu}}\right\} \mid=>\left\{\widehat{\mathbf{M}}_{\mathbf{r}, \boldsymbol{\mu \nu}}, \widehat{\mathbf{M}}_{\mathbf{r}}^{\boldsymbol{\mu \nu}}\right\}$, having M's like Higgs metrics mass of HiggsBoson matter. Equation (4) then will become:

$$
\left(\begin{array}{cc}
\widehat{\varepsilon}_{\mathbf{r}, \mu \nu} & \nabla^{2} \mathbf{E}_{\mathrm{g}}^{\mu \nu} \\
\nabla^{2} \mathbf{E}_{\mathbf{g}, \mu \nu} & \widehat{\boldsymbol{\varepsilon}}_{\mathbf{r}}^{\mu \nu}
\end{array}\right)|=><=|\left(\begin{array}{cc}
\widehat{\mathbf{M}}_{\mathbf{r}, \mu \nu} & \widehat{\mathbf{G}}_{\mathrm{g}}^{\mu v} \\
\widehat{\mathbf{G}}_{\mathrm{g}, \mu \nu} & \widehat{\mathbf{M}}_{\mathrm{r}}^{\mu \nu}
\end{array}\right)
$$

Additionally, per arguments [11], gradient zero-point $\left\{\boldsymbol{\nabla}^{\mathbf{2}} \mathbf{E}_{\mathbf{g}, \boldsymbol{\mu \nu}}, \boldsymbol{\nabla}^{\mathbf{2}} \mathbf{E}_{\mathbf{g}}^{\boldsymbol{\mu \nu}}\right\} \mid=>\left\{\widehat{\mathbf{G}}_{\mathbf{g}, \boldsymbol{\mu \nu}}, \widehat{\mathbf{G}}_{\mathbf{g}}^{\boldsymbol{\mu \nu}}\right\}$ will have Gilbertian nature, and the $\left\{\widehat{\boldsymbol{\varepsilon}}_{\mathbf{r}, \boldsymbol{\mu \nu}}, \widehat{\boldsymbol{\varepsilon}}_{\mathbf{r}}^{\mu \nu}\right\} \mid=>\left\{\widehat{\mathbf{M}}_{\mathbf{r}, \boldsymbol{\mu \nu}}, \widehat{\mathbf{M}}_{\mathbf{r}}^{\boldsymbol{\mu \nu}}\right\}$ will have Amperian nature. We can show that in vacuum gravitational fields, $\widehat{\mathbf{M}}_{\mathbf{r}, \boldsymbol{\mu \nu}}->0$ and $\left\|\widehat{\mathbf{M}}_{\mathbf{r}}^{\boldsymbol{\mu \nu}}\right\| \equiv \mathbf{M}$, representing like the flavor Higgs-Boson matter mass, quantifying inertia with gravitational field manifestations. Wherefore, Equation (5) will become in vacuum gravitational fields:

$$
\left(\begin{array}{cc}
\widehat{\mathbf{M}}_{\mathbf{r}, \boldsymbol{\mu \nu}} & \widehat{\mathbf{G}}_{\mathbf{g}}^{\boldsymbol{\mu \nu}} \\
\widehat{\mathbf{G}}_{\mathbf{g}, \boldsymbol{\mu \nu}} & \widehat{\mathbf{M}}_{\mathbf{r}}^{\boldsymbol{\mu \nu}}
\end{array}\right)|=><=|\left(\begin{array}{cc}
\mathbf{0} & \widehat{\mathbf{G}} \\
\widehat{\mathbf{G}}^{-1} & \widehat{\mathbf{M}}
\end{array}\right)
$$

with $\widehat{\mathbf{G}} \equiv$ gauge, $[\mathbf{G}] ; \widehat{\mathbf{G}}^{-\mathbf{1}} \equiv$ gauge inverter, $[\mathbf{G}]^{-1} ; \widehat{\mathbf{M}} \equiv$ flavor or the dark matter energy, $[\mathbf{M}]$, giving determinant equating $0 . \widehat{\mathbf{M}}-\widehat{\mathbf{G}}^{-\mathbf{1}} \widehat{\mathbf{G}}=0-\mathrm{I}$, having magnitude of -1 . 
We may also rewrite:

$\left(\begin{array}{cc}\mathbf{0} & \widehat{\mathbf{G}} \\ \widehat{\mathbf{G}}^{-\mathbf{1}} & \widehat{\mathbf{M}}\end{array}\right)$ to have unitary determinant $\left(\begin{array}{cc}\mathbf{0} & \widehat{\mathbf{G}} \\ \widehat{-G}^{-1} & \widehat{\mathbf{M}}\end{array}\right)$

so that determinant of this matrix is an identity I, instead of -I. Further, $\widehat{-G}^{-1}$ will represent a point mirror symmetry of $\widehat{\mathbf{G}}$. These aspects will be addressed later in more w.r.t. crystal point mathematical formalism proofs with "Rotation Matrix" in Materials Science Group Theory, deriving formalism with point matrix reflection imaginary parity value.

It can also be noted by comparison with physics literature these results have similarity or an analogous model reflective of non-Hermitian quantum CPT physics [25]. We may also note that this matrix in Equation (5.2) can represent PT symmetry with quaternion typically having typical "-1 problem" [25-30]. In subsequent papers, author will highlight analyzing these aspects. What the above foregoing results and discussions analytically project will be capability to have extension of gauge matrix metrics, Equation (5.2) like stringmetrics, shown below.

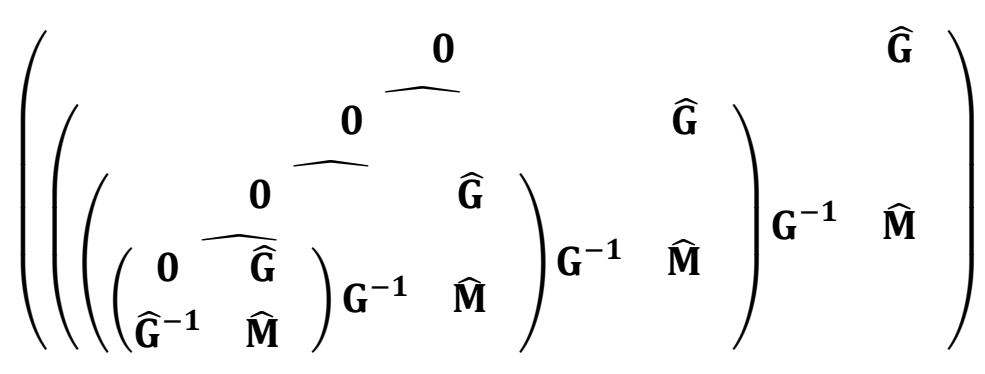

Graphic Figure Equation (6) Matrix construct showing charge asymmetry gage metrics key.

Essentially, $\widehat{\mathbf{G}}^{\mathbf{- 1}} \mathbf{-} \widehat{\mathbf{G}}$, cross-diagonal, will extend like gage "ray" analogous to negative charge like fermion or electron of Gilbertian nature at infinity bringing to real spacelike volt or potential; it is like classical definition of potential unit volt. However, non-gauge with $\widehat{-\mathbf{G}}^{-1}$ also refer to point mirror symmetry of $\widehat{\mathbf{G}}$, as pointed out earlier, which is adequately considerable as gauge field having "star ray". In essence, therefore $\widehat{\mathbf{G}}^{\mathbf{- 1}} \mathbf{-} \widehat{\mathbf{G}}$ will represent Coulomb gage fermion charge of microblackhole at infinity of vacuum to real space gauge field of radiation wave. These aspects link charge conjugation to rotating charges per Dirac Maxwell Einstein Kerr Newman metrics [23, 24].

Whereas 0 -> $\widehat{\mathbf{M}}$, the diagonal Hilbert Higgs metrics within physics literature [22], perhaps quantifying Higgs mechanistic field operator generator, signifying action to matter inertia effectively operating with gravitational field moving from vacuum to matter, $\mathbf{M}$ in general will represent Helmholtz transformation symplectics to Higgs field, having subsequent Higgs mechanism to originate God particle giving flavor mass particle Higgs Boson system [11, 12,2129].

Now turning our attention to application of matrix process general formalism having Equation (11) [13]: $\mathrm{F}_{\mathrm{t}}^{\mathrm{E}}=\boldsymbol{\rho}(\mathrm{t})\left(\left\langle\boldsymbol{\Psi}_{\mu}(\mathrm{t}) \mid \boldsymbol{\Psi}^{\mu}(\mathrm{t})\right\rangle\right)^{-\mathbf{1}} \mathbf{V}$, we substitute equivalent gage parameters, 
obtained from above rigorous derivations as: $\boldsymbol{\nabla}^{\mathbf{2}} \mathbf{E}_{\mathbf{g}}^{\boldsymbol{\mu \nu}}=\widehat{\mathbf{G}}$, quantifying in Equations (5) and (6), we can write logically conclusively extending above solution's arguments as follows:

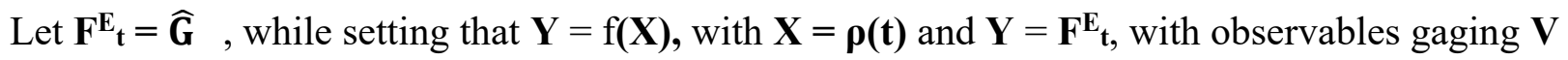
$=\left\|\boldsymbol{\nabla} \quad \mathbf{E}_{\mathbf{g}}^{\mu v}\right\|$, giving scalar potential, working with $\boldsymbol{\rho}(\mathbf{t})=$ quantum density matrix, typically representing pure state like coupling constant with general relativity. So,

$$
\widehat{\mathbf{G}}=\left(<\Psi_{\mu}(\mathbf{t}) \mid \Psi^{\mu}(\mathbf{t})>\right)^{-1}\left\|\nabla \quad E_{g}^{\mu v}\right\| \rho(t)
$$

$\mathrm{f}$, function operator transforming micro to macro parametrically $\boldsymbol{\rho}(\mathbf{t})$ to $\widehat{\mathbf{G}}$ is quantifiable like: $\mathrm{f}=\left(\left\langle\left.\Psi_{\mu}(\mathrm{t})\right|^{\mu} \boldsymbol{\mu}(\mathbf{t})>\right)^{-1}\left\|\nabla \quad \mathbf{E}_{\mathrm{g}}^{\mu \nu}\right\|\right.$.

With quantum mechanics extendable to non-Hermitian and hydrodynamical precessions classical observables quantum physics considerations [25-36], it is possible to advance general gage formalism quantified above to more compact algorithm of switching modes, that may provide most fundamental physics with nature [37]. In terms of typical systems switching modes $\{0$, off, on $\}[37]$, functor can mathematically characterize existence of physics 0 mode $=>::<=$ on_mode. Off_mode switches may provide compatible coupling function activating functional from 0_mode towards on_mode; similarly, off_mode coupling function transitioning from on_mode towards $0 \_$mode can also provide conjugately compatible coupling function activating functional. These processes thence can be effective causality to quantum entangle categorical mode states; we may also note that by virtue of typical character of a functor $[15,18]$, physically 0 and on modes are quantum decohered at their default states.

One can exemplify observables' analogies with the logical comparable statements giving: rock is like 0_mode, and fire is like on_mode; then, air is like off_mode function that if it can be compatible coupler, then it may activate quantum entanglement of 0 and on modes' default decohered states with natural processes phenomena. Testing of the physical observables naturally may be evident from observation of rock fires, that require special configuration of air with pressure, temperature, humidity, environmental chemistry, among other properties and aspects to get activated epiphany.

These formal gage metrics set proper situations to apply unitarization processes that help in gage matching special unitary group mathematical physics. They may then properly herald a grand unification scheme to bring together Theory of Everything, Grand Unified Theories, Standard Model, String Theories, Super Symmetry, Quantum Field Theory with charge, parity, time reversal physical resolutions of matter antimatter asymmetry and provable observables with physics natural phenomena processes. The present question about the role of monopoles will highlight author's ongoing collaborative physics projects with international scientific groups.

\section{Physical Analysis with Results and Discussions}




\subsection{Brief note about Helmholtz metrics gage to axial eccentric quantum fields gravity}

With our previous section 2.1 discussion of gage inverter with gage, we continue analyzing $\widehat{\boldsymbol{G}}^{\mathbf{- 1}}->\widehat{\boldsymbol{G}}$ gage "ray" as being nonorthogonal to $0->\widehat{\boldsymbol{M}}$ vacuum gravitational field axially, having also the diagonal-cross_diagonal matrix with eccentric gage "ray" axiality. This promotes precessions, like well-known classical angular momenta physics, as well as nuclear magnetic resonance [31-33]. Earth's seasons are evolved from precession around eccentric axis [34-35]. Hence these are fundamental consequence of magneto gravitational effect out of above gage matrix, Equation (6), that can explain Coulomb Gilbertian magnetic monopole gage non-gage axial precession around Hilbert Amperian vacuum gravitational field due to nonorthogonal axialities. Quite possibly, microscopic quantum time reversibility inferable from physics with Equation of Gage Strings (6), giving local inversion with non-gage matter $\widehat{\boldsymbol{G}}^{-\mathbf{1}}->\boldsymbol{M}<-\widehat{\boldsymbol{G}}^{-\mathbf{1}}$ gravitational interactive tunneling collapse effect may be analyzed further. Also, one may surmise that these may lead to time compression that has proper potential in shifting relativistic worldlines [36].

\subsection{Superposing Signal Superluminal Profile Graph onto Proper Real Extension Time}

Like transparencies overlaying on original signal graph [12], one can mathematically hereby conceive of stacking 5 or 6 Riemann sheets like planes, possibly overlaying typically Riemann sheets with Planck thickness " $h$ " [39-41]. Presently author will enumerate following listing only to explore later:

(i) constructing on "Vacuum Genesis" original physics literature c speed of light graphic, how real time and proper time will act like outliers.

(ii) superluminal signal profile graph [12] dipping onto outliers' real proper time.

(iii) $\quad \widehat{\mathbf{G}}^{-\mathbf{1}} \rightarrow \mathbf{M}<-\widehat{\mathbf{G}}^{-\mathbf{1}}$ extended matrix with constructing Coulomb gage out of Helmholtz matrix already demonstrated having above Equation (6) to generalize unification theories.

(iv) 0-off-on switching modes, with "off" switching mode at left edge having the $\mathbf{- \mathbf { G } ^ { - 1 }}$ and "on" switching mode at the right edge of the $\widehat{\mathbf{G}}$ gage ray, that is nonorthogonal to the $0->\widehat{\mathbf{M}}$ vacuum gravitational field axial, and diagonal-cross diagonal matrix with eccentric gage ray axiality. Notably, [0, off, on] modes will satisfy "Theorem of Switches" [37]. $\widehat{\mathbf{M}}$ will then add as the fourth "diamond" bridge to the gravitational field proper time metrics branch, indicating non-linear time pathing worldline. 
(v) Quantum Field Theory Feynman diagram [38] also superposable with having vertically twisted or equivalently phase shifted diagram, pointing positron and electron pairs correct direction to the gage signal graph, with branching of light or magneto field originating from monopole to electron positron generator, considered more here in Particle Physics discussions.

\subsection{Particle Physics Gage Matrix}

Equation (6) may be inferred as pointing to a typical Dirac's Sea of electrons towards left infinity of $\widehat{-G}^{-1}$ Dirac monopoles, while generating positrons at the right infinity of $\widehat{\mathbf{G}}$, along the gage ray, $\widehat{\mathbf{G}}^{\mathbf{- 1}} \rightarrow>\widehat{\mathbf{G}}$. This separation of the charges can be thought to be likely generators of the spatial matter. It is also quite interesting that local inversion of gage-nongage, $\widehat{\mathbf{G}}^{-\mathbf{1}} \rightarrow \mathbf{M}$ $<-\widehat{\mathbf{G}}^{-1}$ will constitute positron electron annihilation, leading to energy production, relativistically energy, $\mathrm{E} / \mathrm{c}^{2}=\mathrm{M}$, will generate matter as well. Quantum relativity physics becomes consistent theory of everything thus. The vortex Helmholtz decomposition fields may play role of possibly converging thereby generating compressed monopoles, for example, or may expand after divergence to create vacuum monopoles, as expounded below. We analogically equate zero-point $\equiv$ vacuum monopole, microblackhole $\equiv$ gravitational monopole, particle $\equiv$ discrete entity of partial differential equations. In the very "cradle" of the "curdling" plane, $\widehat{\boldsymbol{G}}^{\mathbf{- 1}}->\boldsymbol{M}<-\widehat{\boldsymbol{G}}^{\mathbf{- 1}}$ can to the $\mathrm{E} / \mathrm{c}^{2}=\mathrm{M}$ transformation to create typically neutrino antineutrino pair orthogonal to electron positron "curdling" plane. Far out in that action region, electron + neutron, synthesized out of neutrino grouping, may give rise to proton, which is a hydrogen $\mathrm{H}+$, that are the starry matter. In contrast, away from it (orthogonally), positron + antineutrino may give rise to muons, which constitute equivalent electrons' mass that much lower. Hence one physics conjecture possibly is that "curdling" of the milky way having Equation (6) of particles with "galaxy" referring to the high signal profile of superluminal velocity [12] will constitute plasmatic quagmire. These are explainable in the context of quantum astrophysics given below.

\subsection{Quantum ASTROPHYSICS Gage Matrix}

Equation (6) as well as this superluminal profile of signals velocity [12] may provide more insight with local inversion of gage-nongage, $\widehat{\mathbf{G}}^{\mathbf{- 1}}->\mathbf{M}<-\widehat{\mathbf{G}}^{\mathbf{- 1}}$ constituting electron positron annihilation, leading to energy production, having relativistically energy originating matter per that $\mathrm{E} / \mathrm{c}^{2}=\mathrm{M}$. We know per quantum field theory that electron positron can generate chain like "curdling" action, as per physics literature, explained above in Section 4. Hence one physics conjecture, as mentioned above is possibly that "curdling" of the milky way having Equation (6) of particles with "galaxy" referring to the high signal profile of superluminal velocity [12] will constitute plasmatic quagmire. Overall, receding or fast expanding universe with dark matter may be happening in the background as well that will emphasize about missing matter paradox. One may think of $\boldsymbol{M}$, the flavor metrics with the Standard Model in general with $\boldsymbol{G}$, the gage 
field associating with metric flavor. Author is pursuing these aspects of Quantum Astrophysics with his scientific group, where a theoretical model Einstein Gauss Bonnet gravity is fully developed alongside quantum physical analyses that will explain observations of dark energy stars [42].

\subsection{Interpretations on virtual gravitational dipoles:}

One may also construe Equation (6) $\widehat{\mathbf{G}}^{-\mathbf{1}}->\mathbf{M}<-\widehat{\mathbf{G}}^{-\mathbf{1}}$ to be two different representations of the gravitational monopoles having gravitational charge of opposite sign per physics literature [24-26. 29]. Gravitational monopoles probably occur at Planck dimensions particularly originating center of a supermassive blackhole $[43,44]$. While vacuum monopoles probably occur infinitely with universal vacuum quanta cosmos extent $[45,46]$.

\section{Summary conclusions}

Author has presented convincingly general formulation of gaging Helmholtz Hamiltonian mechanics to Amperian Gilbertian Hilbert Coulomb Matrix Gage fields electromagnetic gravity. Iyer Markoulakis Helmholtz Hamiltonian general formalisms have been aptly converted to gage matrix, following physics literature procedures. Transformation of Helmholtz metrics to Coulomb gage, linking also Coulomb branch gage group with Hilbert series has been quantifiably achieved having gradient fields $\left\{\boldsymbol{\nabla}^{\mathbf{2}} \mathbf{E}_{\mathbf{g}, \boldsymbol{\mu \nu}}, \boldsymbol{\nabla}^{\mathbf{2}} \mathbf{E}_{\mathbf{g}}^{\boldsymbol{\mu \nu}}\right\} \mid=>\left\{\widehat{\mathbf{G}}_{\mathbf{g}, \boldsymbol{\mu \nu}}, \widehat{\mathbf{G}}_{\mathbf{g}}^{\mu \boldsymbol{v}}\right\}$ Coulomb gage, with branching to Hilbert gauge rotational vortex fields $\left\{\widehat{\boldsymbol{\varepsilon}}_{\mathbf{r}, \boldsymbol{\mu \nu}}, \widehat{\boldsymbol{\varepsilon}}_{\mathbf{r}}^{\boldsymbol{\mu \nu}}\right\} \mid=>\left\{\widehat{\mathbf{M}}_{\mathbf{r}, \boldsymbol{\mu \nu}}, \widehat{\mathbf{M}}_{\mathbf{r}}^{\mu \boldsymbol{\nu}}\right\}$, having M's like Higgs metrics mass of Higgs-Boson matter, and conforming to partial differential equations of vortex and the gradient fields obtained per Iyer Markoulakis original formalism.

Vacuum gravitational solutions of the fields provided means to arrive at unitary $\operatorname{determinant}\left(\begin{array}{cc}\mathbf{0} & \widehat{\mathbf{G}} \\ \widehat{-G}^{-1} & \widehat{\mathbf{M}}\end{array}\right)$ that will analytically project having extension of gauge matrix metrics like stringmetrics construct showing charge asymmetry gage metrics:

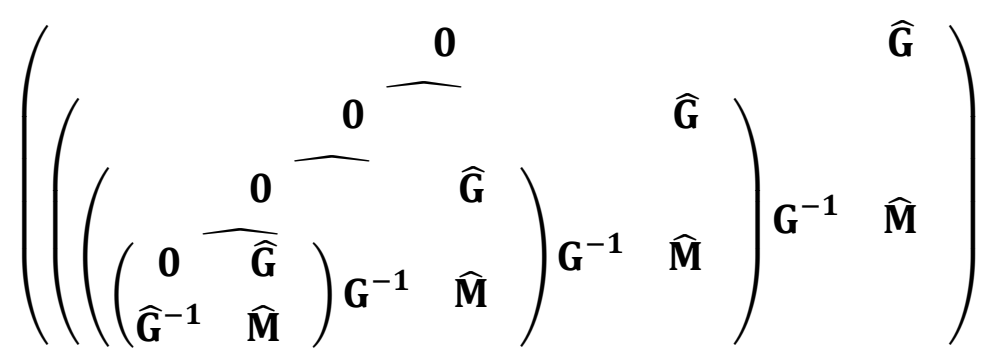


$\widehat{\mathbf{G}}^{\mathbf{- 1}} \mathbf{-} \widehat{\mathbf{G}}$, cross-diagonal can be extended like gage "ray" analogous to negative charge like a Standard Model having a fermion or electron of Gilbertian nature at infinity bringing to real spacelike volt or potential, while non-gauge with $\widehat{-\mathbf{G}}^{-1}$ refer to point mirror symmetry of $\widehat{\mathbf{G}}$, typically. Whereas $0->\widehat{\mathbf{M}}$, the diagonal Hilbert Higgs metrics can quantify as Higgs mechanistics field operator generator, signifying action to matter inertia in terms of gravitational field moving the vacuum to matter, $\mathbf{M}$ in general representing Helmholtz transformational symplectics to typical Higgs field, having subsequent Higgs mechanism to originate God particle giving flavor mass particle Higgs Boson system.

Further basing author's recent proof formalism, invoking prime matrix properties, derivation of a general potential wave quantum density commutator matrix physics, gage equivalent expressions have been advanced like $\widehat{\mathbf{G}}=\left(\left\langle\left.\boldsymbol{\Psi}_{\boldsymbol{\mu}}(\mathbf{t})\right|^{\boldsymbol{\mu}}(\mathbf{t})\right\rangle^{-\mathbf{1}}\left\|\boldsymbol{\nabla} \quad \mathbf{E}_{\mathrm{g}}^{\mu \boldsymbol{v}}\right\| \boldsymbol{\rho}(\mathbf{t})\right.$, incorporating observables gaging $\mathbf{V}=\left\|\boldsymbol{\nabla} \quad \mathbf{E}_{\mathbf{g}}^{\mu \boldsymbol{v}}\right\|$, scalar potential combining with $\boldsymbol{\rho}(\mathbf{t})=$ quantum density matrix, typically representing pure state, like coupling constant in general relativity. Observables' analogies statements compact algorithm of switching modes characterizing most fundamental physics with nature: rock is like $0 \_$mode, and fire is like on_mode; then, air is like off_mode function that maybe epiphany evident from observation of rock fires.

Physical analysis explaining Helmholtz metrics gaging to axial eccentric quantum fields gravity, superimposing model of signal superluminal profile Graph onto proper and real extended time gage, particle physics gage matrix to model a way to quantify Helmholtz Hamiltonian mechanics to Dirac monopoles, quantum astrophysics gage matrix conceptualizations, formalism applying to interpretations on virtual gravitational dipoles, vacuum, and gravitational monopoles at Planck dimensions.

Author is already working to have verification of physical observables with experimental physicists, that will also span quantum, mesoscopic, astrophysical quantum relativistic and classical consistency within a material environmental system having order of magnitude validity, thus narrowing the current range of trillions differential magnitude existing presently.

Fundamental logical physical mathematics abstracting observable phenomena mechanisms provide key to physics natural quantifiability that these formalisms demonstrate adequately. Gage metrics set proper situations to apply unitarization processes that help in gage matching special unitary group mathematical physics will be next step that author is hoping to undertake. Author hopes having applications spanning across many branches of science, engineering, technology, algorithmic mathematics application to Information Systems, specifically extending to artificial expert systems with quantum computing physics.

\section{Acknowledgements}

Encouragements with group projects support of Emmanouil Markoulakis of Hellenic Mediterranean University in Greece, Manuel Malaver of Maritime University of the Caribbean 
in Venezuela, and perpetual project support of Engineeringinc International Operational Teknet Earth Global Platform collaborations would be appreciated highly. Author is thankful particularly to active RESEARCHGATE scientific physics forums to stimulate sessions providing collaborative ongoing debates, having brain storming progress.

\section{References}

[1] Frank Wilczek: Physics in 100 years, Physics Today, Volume 69, Issue 4, 2016, https://physicstoday.scitation.org/doi/10.1063/PT.3.3137.

[2] Frampton, P. (2008). Gauge Field Theories (3rd ed.). Wiley-VCH.

[3] Becchi, C. (1997). "Introduction to Gauge Theories". arXiv:hep-ph/9705211.

[4] Jackson, J.D. (2002). "From Lorenz to Coulomb and other explicit gauge transformations". Am. J. Phys. 70 (9): 917-928. arXiv:physics/0204034. Bibcode:2002AmJPh..70..917J., doi:10.1119/1.1491265.

[5] Svetlichny, George (1999). "Preparation for Gauge Theory". arXiv:math-ph/9902027.

[6] Stefano Cremonesi, Amihay Hanany, Noppadol Mekareeyab, and Alberto Zaffaronic:

Coulomb branch Hilbert series and Hall-Littlewood polynomials, Journal of High Energy

Physics, Volume 2014, article id.178, 59 pp., arXiv:1403.0585, DOI:10.1007/JHEP09(2014)178.

[7] CLASSICAL GAUGE FIELDS, ch.3 of Field Theory, https://www.reed.edu/physics/faculty/wheeler/documents/Classical\%20Field\%20Theory/Class\% 20Notes/Field\%20Theory\%20Chapter\%203.pdf.

[8] Eduardo I. Guendelman, Douglas Singleton, "Scalar gauge fields", Journal of High Energy Physics, 2014 - Springer, JHEP 1405 (2014) 096, doi:10.1007/JHEP05(2014)096.

[9] Lisa Randall, "New Mechanisms of Gauge-Mediated Supersymmetry Breaking”, High Energy Physics, Nuclear Physics - Phenomenology Theory, Section B, 495, 1, 37-56, 1997 , arXiv:hep-ph/9612426, https://doi.org/10.1016/S0550-3213(97)00225-3; application to special unitary group supersymmetric mass terms.

[10] Emmanouil Markoulakis, Antonios Konstantaras, John Chatzakis, Rajan Iyer, and Emmanuel Antonidakis, "Real time observation of a stationary magneton", Results in Physics, 2019, Vol. 15, 102793, https://doi.org/10.1016/j.rinp.2019.102793.

[11] Rajan Iyer, and Emmanouil Markoulakis, "Theory of a superluminous vacuum quanta as the fabric of Space", accepted publication with PHYSICS \& ASTRONOMY INTERNATIONAL JOURNAL, 2021.

[12] Rajan Iyer, "Problem Solving Vacuum Quanta Fields", International Journal of Research and Reviews in Applied Sciences, Volume 47, Issue 1, PP. 15-25, 2021. 
[13] Rajan Iyer, "Proof Formalism General Quantum Density Commutator Matrix Physics", publication in progress.

[14] Jean Zinn-Justin and Riccardo Guida, "Gauge invariance”, Scholarpedia, 3(12):8287, 2008, http://www.scholarpedia.org/article/Gauge_invariance.

[15] https://ncatlab.org/schreiber/show/Course+on+stacks+and+action+functionals.

[16] "Gauge Invariance of the Hamiltonian of the electromagnetic field", https://physics.stackexchange.com/questions/94699/gauge-invariance-of-the-hamiltonian-of-theelectromagnetic-field.

[17] Schwarz, Albert (2000). "Topological quantum field theories". arXiv:hep-th/0011260.

[18] Anders K. H. Bengtsson, "Structure of higher spin gauge interactions”, Journal of Mathematical Physics 48, 072302, 2007, https://doi.org/10.1063/1.2751277.

[19] Anders K. H.Bengtsson, “Towards Unifying Structures in Higher Spin Gauge Symmetry?", Symmetry, Integrability and Geometry: Methods and Applications, SIGMA 4, 013, 23 pages, 2008, http://www.emis.de/journals/SIGMA/2008/013/, or https:/www.emis.de/journals/SIGMA/2008/013/sigma08-013.pdf.

[20] V. Fock, Z. Phys. 75 (1932), 622-647] M.C. Reed, B. Simon, "Methods of Modern Mathematical Physics, Volume II", Academic Press 1975. Page 328. Also refer: Wikipedia.

[21] Kirk T. McDonald, “The Helmholtz Decomposition and the Coulomb Gauge”, Joseph Henry Laboratories, Princeton University, Princeton, NJ 08544, April 17, 2008; updated March 3, 2020, https://physics.princeton.edu// mcdonald/examples/helmholtz.pdf.

[22] Guillermo Arias-Tamargo, Antoine Bourget, Alessandro Pini, Diego Rodríguez-Gómez, "Discrete gauge theories of charge conjugation", Nuclear Physics B, Volume 946, September 2019, 114721, https://www.sciencedirect.com/science/article/pii/S055032131930207X https://reader.elsevier.com/reader/sd/pii/S055032131930207X?token=25C397DE4643005A0E2 77BFD0FEB90EEA3AF961776A4EEE6F086FCB908466D3D3F087E1FE949AB95827A4D67 B8C8D249.

[23] Elena Giorgi, "The Carter tensor and the physical-space analysis in perturbations of KerrNewman spacetime", General Relativity and Quantum Cosmology, Mathematical Physics Analysis of PDEs, arXiv:2105.14379, 2021.

[24] Dietrich Hafner, and Jean-Philippe Nicolas, "Scattering of Massless Dirac Fields by a Kerr Black Hole", Reviews in Mathematical Physics, Vol. 16, No. 01, pp. 29-123, 2004, https://doi.org/10.1142/S0129055X04001911, https://www.worldscientific.com/doi/epdf/10.1142/S0129055X04001911.

[25] Steven Weinberg: Lectures on Quantum Mechanics - 2nd Edition, 2013, Cambridge University Press, www.amazon.com. 
[26] Katherine Jones-Smith, about identifying quasi-particles using non-Hermitian quantum mechanics, pp. 1-14, using PT quantum mechanics, Philosophical Transactions: Mathematical, Physical and Engineering Sciences, Vol. 371, No. 1989, 2013, Published by: Royal Society, https://www.jstor.org/stable/i40090204.

[27] Sabine Hossenfelder, "Interpretation of Quantum Field Theories with a Minimal Length Scale", Phys. Rev. D, 73, 105013, 2006, arXiv:hep-th/0603032, https://doi.org/10.1103/PhysRevD.73.105013.

[28] Matthew D. Schwartz: Quantum Field Theory and the Standard Model, 2014, Cambridge University Press, www.amazon.com.

[29] Paul Adrien Maurice Dirac, "Quantised singularities in the electrovacuum field", Proc. R. Soc. Lond., A13360-72, http://doi.org/10.1098/rspa.1931.0130.

[30] Carl M. Bender: PT Symmetry: In Quantum and Classical Physics, WORLD SCIENTIFIC PUBLISHING, 2019, Amazon.com.

[31] David Halliday, Robert Resnick, and Jearl Walker: Fundamentals of Physics: Extended, 11th Edition, 2018, 1456 pages, WileyPLUS Publishing, ISBN: 978-1-119-30685.

[32] David Milstead: The Quantum Mechanics of MRI, https://www.yumpu.com/en/document/ view/20968059/the-quantum-mechanics-of-mri-part-1-basic-concepts.

[33] Robert Eisbeg: Quantum Physics of Atoms, Molecules, Solids, Nuclei, and Particles, Second Edition, 1985, ISBN 0-471-87373-X, John Wiley \& Sons.

[34] Van den Heuvel EP, "On the Precession as a Cause of Pleistocene Variations of the Atlantic Ocean Water Temperatures", Geophysical Journal International, 11 (3): 323-336, 1966, doi:10.1111/j.1365-246X.1966.tb03086.x.

[35] A. Berger, M. F. Loutre, and J. L. Mélice, "Equatorial insolation: from precession harmonics to eccentricity frequencies" (PDF), Clim. Past Discuss., 2 (4): 519-533, 2006, DOI:10.5194/cpd-2-519-2006.

[36] Andrew J. S. Hamilton: General Relativity, Black Holes, and Cosmology, https://jila.colorado.edu/ ajsh/astr3740_17/grbook.pdf.

[37] Rajan Iyer: Absolute Genesis Fire Fifth Dimension Mathematical Physics, 2000, Engineeringinc International Publisher with Amazon.com.

[38] David Kaiser, "Physics and Feynman's Diagrams", American Scientist, 93 (2): 156, 2005, doi:10.1511/2005.52.957.

[39] Joanna D. Haigh (Referee), "Interactive comment on "The representation of solar cycle signals in stratospheric ozone. Part II: Analysis of global models" by Amanda C. Maycock et al.", Atmos. Chem. Phys. Discuss., https://doi.org/10.5194/acp-2017-477-RC1, 2017, Atmospheric Chemistry and Physics, ISSN: 1680-7316. 
[40] K. A. Michalski and M. M. Mustafa: "On the computation of hybrid modes in planar layered waveguides with multiple anisotropic conductive sheets”, Proc. R. Soc. A, 474: 20180288, 2018, http://dx.doi.org/10.1098/rspa.2018.0288.

[41] Giampiero Passarino, Christian Sturm, and Sandro Uccirati, "Higgs Pseudo-Observables, Second Riemann Sheet and All That”, Nucl.Phys.B, 834, 77-115, 2010, DOI:

10.1016/j.nuclphysb.2010.03.013.

[42] Manuel Malaver, Hamed Daei Kasmaei, Rajan Iyer, Shouvik Sadhukhan, and Alokananda Kar, "A theoretical model of Dark Energy Stars in Einstein-Gauss-Bonnet Gravity", accepted for publication in Applied Physics, 2021, arXiv:2106.09520.

[43] I. M. Benn, T. Dereli, and R W Tucker, "Gravitational monopoles with classical torsion”, Journal of Physics A: Mathematical and General, Volume 13, Number 10, L359.

[44] Y. M. Cho, "Theory of Gravitational Monopole", https://inspirehep.net/files/5715e99ae0c602c5917424efaea76e7b.

[45] L. D. Lantsman and V. N. Pervushin, "Monopole Vacuum in Non-Abelian Theories", Phys. Atom.Nucl., 66, 1384-1394, 2003, arXiv:hep-th/0407195, DOI:10.1134/1.1592593.

[46] Sarah Tesh, "Flash Physics: Sandra Faber wins Gruber prize, transforming magnetic monopoles, vacuum scattering revealed", COSMOLOGY NEWS, https://physicsworld.com/a/flash-physics-sandra-faber-wins-gruber-prize-transformingmagnetic-monopoles-vacuum-scattering-revealed.

Sarah Tesh is features editor of Physics World. 